\title{
Un acercamiento a lo radical de la convivencia
}

\section{An approach to the radical of coexistence}

DOI: http://dx.doi.org/10.17981/cultedusoc.9.1.2018.04

Recibido: 12 de diciembre de 2017 / Aceptado: 15 de marzo de 2018

Eduardo Arias Castañeda ${ }^{1}$

Instituto Tecnológico y de Estudios Superiores de Occidente-ITESO (México) arias@iteso.mx

Para citar este artículo

Arias, E. (2018). Un acercamiento a lo radical de la convivencia. Cultura. Educación y Sociedad 9(1), 59-68. DOI: http://dx.doi.org/10.17981/ cultedusoc.9.1.2018.04

\section{Resumen}

En este trabajo, es un recorrido que inicia con algunos antecedentes vinculados a la Organización de la Naciones Unidas para la Educación, la Ciencia y la Cultura (UNESCO) ya que sus propuestas educativas dieron un fuerte impulso al tema de la convivencia, con un enfoque universal, incluyente y con visión de futuro. En segundo momento se aborda el proceso realizado en una investigación realizada en México (Perales, Arias y Bazdresch, 2014) y que da la pauta para visualizar factores convivenciales transversales que tienen lugar en las interacciones humanas del ámbito escolar, las cuales derivan en lo que se nombra como núcleos socio-afectivos. Esta nueva perspectiva permite adentrarnos no solo en la exterioridad del hacer humano, sino también en una reflexión acerca de la vinculación con la corporeidad del ser humano en general. Finalmente se proponen dos temas que pueden orientar las acciones de esta nueva visión: Uno es la ética del cuidado frente a la ética de la responsabilidad; y el otro, el de la "provención" frente a la "prevención", los cuáles nos invitan a ir más allá de una postura estrictamente programática como la que suele establecerse en los centros escolares.

Palabras Clave: Convivencia, paradigma, ética, cuidado, corporeidad.

\begin{abstract}
In this work, it is a journey that begins with some background linked to the United Nations Organization for Education, Science and Culture (UNESCO) as its educational proposals gave a strong impetus to the theme of coexistence, with a focus universal, inclusive and forwardlooking. Secondly, the process carried out in an investigation carried out in Mexico (Perales, Arias \& Bazdresch, 2014) is addressed and it gives the guide to visualize cross-cutting coexistence factors that take place in the human interactions of the school environment, which derive in the which is named as socio-affective nuclei. This new perspective allows us to enter not only into the exteriority of human activity, but also into a reflection on the connection with the corporeity of the human being in general. Finally, two themes are proposed that can guide the actions of this new vision: One is the ethics of care versus the ethics of responsibility; and the other, that of "provention" versus "prevention", which invite us to go beyond a strictly programmatic stance like that which is usually established in schools.
\end{abstract}

Keywords: Coexistence, paradigm, ethics, care, corporeity. 


\section{Introducción}

La convivencia sigue siendo un concepto en construcción que va requiriendo mayor claridad con el fin de lograr acciones educativas más integrales y efectivas en un mundo creciente en complejidad ante los problemas sociales de violencia e inseguridad que enfrentamos tanto a nivel global como a nivel local. En los distintos estudios y análisis en que los investigadores, como se reporta en los estados del conocimiento del COMIE (Furlán \& Spitzer, 2013), el fenómeno de la convivencia muestra diferentes planteamientos y enfoque educativos que, si bien se ordenan hacia propósitos formativos de las relaciones interpersonales en colectividad, también han dejado de lado lo que se puede profundizar en eso que llamamos convivencia en sus aspectos más radicales, de aquello que está inscrito en la propia condición humana. Esto plantea la pregunta acerca de si lo que entendemos por convivencia es solo una construcción social o hay algo más de fondo en ello.

La invitación a visualizar la convivencia desde una perspectiva centrada en la condición humana es resultado de un recorrido de investigación y reflexión a partir del ámbito de la educación. Esto ha permitido ampliar la comprensión del fenómeno y con ello, imaginar nuevas posibilidades de interacción tanto con los otros que nos rodean, así como con la naturaleza misma.

El documento en su conjunto ofrece una nueva ventana al modo de entender la convivencia para aportar nuevos elementos en el vivir la cotidianidad y así fortalecer el tejido comunitario y social.

\section{Un Poco de Historia}

La importancia del tema de la convivencia en el ámbito educativo está vinculado a los últimos informes emitidos por la UNESCO. En orden cronológico son: 1) Aprender a ser. La educación del futuro o también conocido como Informe Faure (UNESCO, 1972). 2) $L a$ educación encierra un tesoro (Delors et al., 1997) al cual se nombró como el informe Delors. 3) El último documento es el que lleva por título: Replantear la Educación. ¿̇Hacia un bien común mundial? (Bokova, 2015). Todos estos documentos, se elaboraron en cada caso, por un grupo de expertos y en todos ellos el trabajo se orientó a responder la pregunta acerca del papel que demanda la educación a nivel mundial. Entre los tres documentos señalados hay una continuidad en el tema de "aprender a ser", pero es en el segundo donde surge la importancia de la convivencia, entendida como el "Aprender a vivir juntos, aprender a vivir con los demás".

Como señala Pérez (2016) con el informe Faure comenzó el cambio de mirada hacia la "Otra Educación" y hacer realidad el sueño de que "Otra Educación es Posible". Una perspectiva que puso la importancia en el individuo cuyo objetivo principal consistió en desarrollar procesos de aprendizajes duraderos tales como "aprender a aprender" y en lograr una actitud de "aprendizaje para toda la vida". Pero el documento más conocido de los tres últimos no deja de ser el "Informe Delors", en honor a su promotor Jaques Delors². En este documento

\footnotetext{
2 Jacques Lucien Jean Delors (París, 20 de julio de 1925), político europeo de nacionalidad francesa. En el año1997 la UNESCO le encargó un estudio sobre la perspectiva de la educación en el mundo de cara al siglo XX.
} 
la Comisión se pregunta: ¿Qué tipo de educación se necesita en el futuro y para qué tipo de sociedad? (Delors et al., 1997); el grupo de expertos que trabajó en esto, enfrentó muchas dificultades para llegar a un acuerdo y poder establecer una base común debido a las diferencias culturales con respecto a cuestiones como: "igualdad frente a jerarquía", "individualismo frente a colectivismo", "cooperación frente a competición" e "idealismo frente a pragmatismo". Fue después de mucho diálogo que se pudo establecer una base común y pronunciar un mensaje universal acerca de la educación. Entre los puntos sobresalientes del informe Delors está el haber optado por una perspectiva humanista, contraria a una educación orientada al mercado, y volviendo a enfatizar el principio de "aprender a ser", el cual constituyó un elemento de continuidad entre el Informe Faure y el informe Delors.

Las diferencias que enfrentó la comisión encabezada por Delors se superaron al centrarse en el concepto de "aprender a convivir", el cual se consideró el más importante de los "cuatro pilares de la educación” y el principio rector del informe (Delors et al., 1996, y Carneiro y Draxler, 2008). Con esto se proponía una visión amplia de la educación donde cada persona pudiera descubrir el tesoro escondido en cada uno de nosotros y trascender una condición instrumental de la educación acotada por intereses principalmente económicos y de mercado. Años más tarde, en sus Memorias, Delors (2005) señaló que "aprender a vivir juntos" (tercero de los cuatro pilares que se abordan ${ }^{3}$ )

${ }^{3}$ El orden que se presenta en el informe es el siguiente: Aprender a conocer, Aprender a hacer, Aprender a vivir juntos, aprender a vivir con lo demás y Aprender a ser. era el más importante de los cuatro y sería en el futuro, la fórmula más contundente para enfrentar los desafíos del mundo que en ese entonces se vislumbraba que íbamos a vivir.

En los países latinoamericanos la influencia del informe Delors no ha sido menor y su importancia queda también explicada por las situaciones que viven estos países y que han dado lugar a crecientes problemáticas socio-culturales, políticas y económica que se manifiestan como violencia, corrupción, desequilibrado reparto de la riqueza, disminución de los recursos planetarios, entre otras. Ante estos fenómenos, las acciones educativas se han orientado en vincular la convivencia con temas de valores, disciplina, justicia, clima escolar, paz, respeto, derechos, tolerancia, diversidad, etc. El avance ha sido significativo en cuanto a la elaboración de investigaciones acerca de la convivencia, así como indicadores, que permiten evaluar los logros en este tema. Sin embargo, el camino por recorrer sigue siendo aún inicial y "aprender a vivir juntos" sigue siendo "una utopía necesaria", como lo expresó Delor en su informe (1996, p. 7).

\section{Otro modo de ver la convivencia}

La noción de lo que se entiende por convivencia sigue apareciendo en algunos momentos como algo exclusivo de los países latinoamericanos debido que el término carece traducción en otros idiomas, Esta diferenciación, que bien puede tener fundamentos históricos de carácter cultural adquiere sentido en la medida que ayuda a entender y a dimensionar la universalidad del 


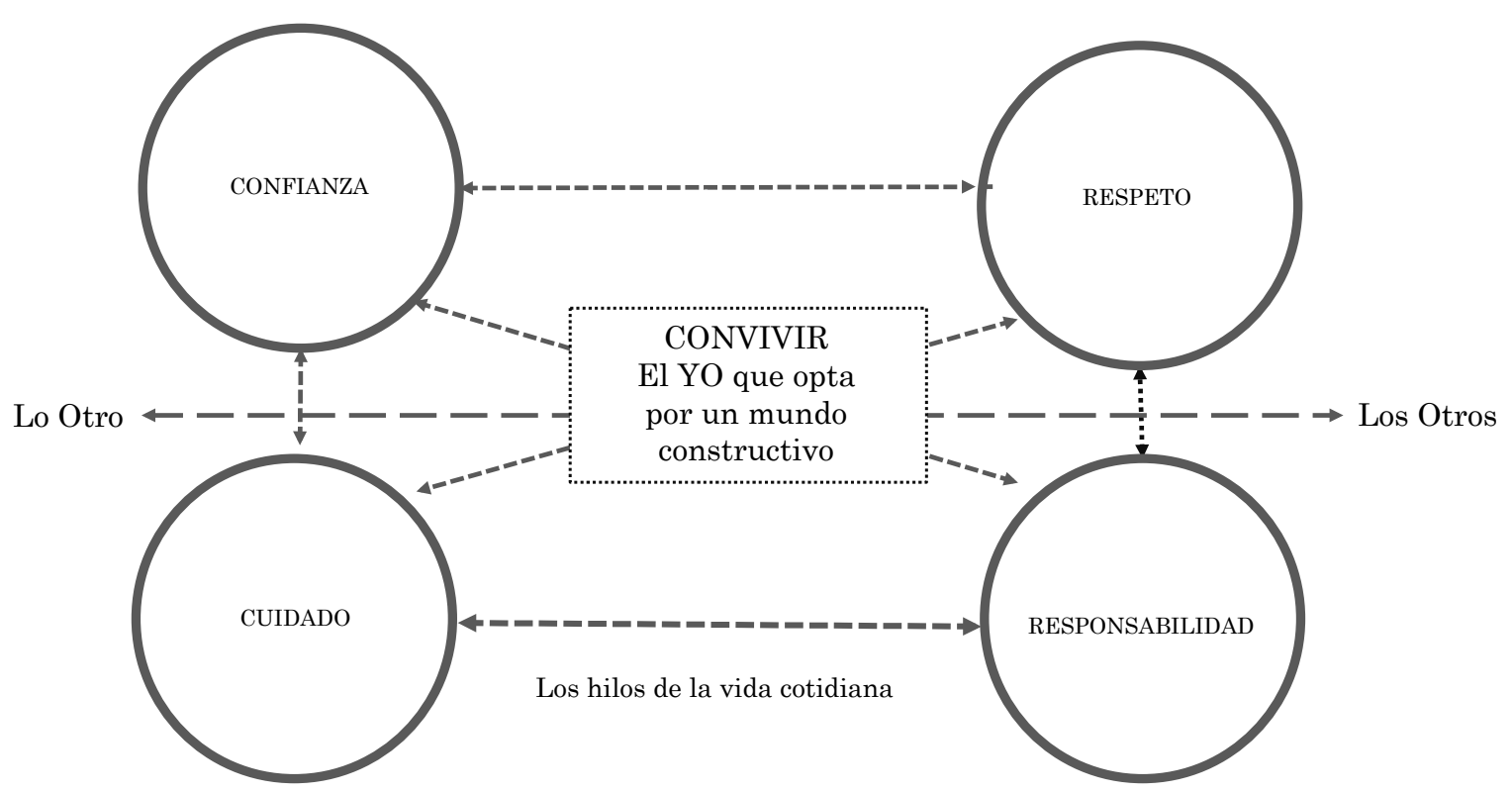

Fig. 1. Núcleos Socio-afectivos

convivir. El término "convivencia" no deja de tener una implicación multicultural cuando la vinculamos al saber "vivir con otros", ya que la unidad de sentido entre una noción y otra radica más en "con" del "vivir, permitiéndonos el uso alternado del término a lo largo de este documento.

Lo importante es que la convivencia es posible visualizarla como un tema a trabajar a fondo para su mejor puesta en práctica y a ser considerada fuente de aprendizajes para la vida y con ello aprender a ser. Esto ha dado pauta para subrayar la importancia del enseñar y aprender a convivir" en los distintos niveles educativos y centros escolares dando lugar a generar políticas institucionales orientadas la convivencia escolar ${ }^{4}$

${ }^{4}$ Se han desarrollado políticas de convivencia escolar en Chile, Argentina, Colombia entre otros países de Latinoamérica y el Caribe a través de sus Ministerios o Secretaría de Educación.
En una investigación realizada en México (Perales et al., 2014) sobre indicadores de convivencia escolar a nivel de educación básica se partió en un principio de visiones aceptadas en diversos estudios y vinculadas a la convivencia. Este enfoque incluyó tres: democracia, inclusión y cultura de paz. A lo largo del proceso se planteó la pregunta acerca de la posibilidad de visualizar elementos de carácter trasversal que evitaran segmentar la visión del "aprender a vivir con otros" o terminaran siendo una mera yuxtaposición de orientaciones. El proceso de análisis y sistematización permitió validar cuatro, a los que se les nombró núcleos socio-afectivos de: confianza, respeto, cuidado y responsabilidad (Figura 1) y referidos todos ellos a las acciones $\mathrm{y}$ actuaciones que tienen lugar en la vida cotidiana. 


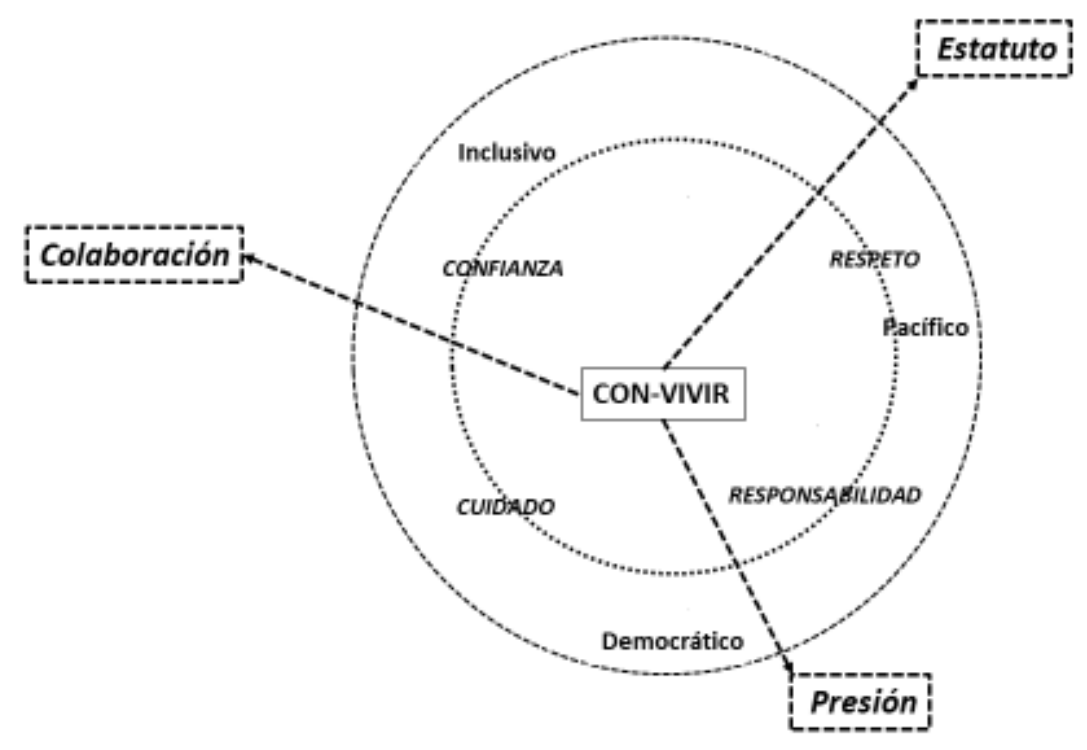

Fig. 2. Fundamentos de la Convivencia

Este nuevo enfoque permitió dar un paso más hacia aspectos menos explorados de la convivencia, pero todavía sin llegar a develar lo más radical de la misma, ya que los factores socio-afectivos no dejan de ser también construcciones que derivan de nuestras interacciones socioculturales. Son acciones que a través de la repetición se convierten en hábitos que dan lugar a una estabilidad en el día al día y permiten tejer con más fuerza y estabilidad las relaciones personales.

\section{Los límites de los enfoques abordados}

El conjunto de elementos considerados constitutivos de la convivencia, se puede agrupar en tres grandes categorías basados en una trabajo elaborado por Xavier Zubiri (2015). Estas categorías son: Colaboración, Estatuto y Presión (Figura 2), pero como se verá más adelante y como lo señala el mismo autor, ninguna termina por dar respuesta a qué es lo radical de la convivencia.

Los límites de estas categorías que explican de la siguiente manera:

\section{- En cuanto a la colaboración}

Al decir que la convivencia es colaboración nos estamos refiriendo a un sistema de relaciones en donde unos colaboran con otros. Sin embargo, la convivencia no es primariamente colaboración, ya que solo hay colaboración cuando el yo laborante está vertido a los otros y dispuesto a convivir con ellos. En otras palabras, el "con" de la convivencia es anterior a la "labor". Solo hay labor y colaboración cuando hay un "con” pero no antes (Zubiri, 2015, pp. 47-48).

\section{- En cuanto estatuto}

Los estatutos son medios institucionales con los cuáles, los individuos construyen un modo de convivencia. A lo largo de la historia se han dado distintas manifestaciones; uno de ellos es el contrato de Rouseau (2007) y otro el de Hobbes (1994). En esta visión de la convivencia hay un modo de vivencia y con ello una cesión para poder lograr el mínimo daño. Las 
constituciones de los países son también estatutos de "con-vivencia" que se expresa como un contrato o concurrencia para hacer posible que cada persona se encuentre con los demás y pueda estar vertido hacia ellos. Visto así, todo estatuto es posterior a la convivencia ya que todo estatuto y toda constitución presuponen la convivencia (Zubiri, 2015, pp. 48-49).

\section{- La convivencia como presión}

En el siglo XIX se veía que la convivencia era esencial y formalmente algo forzoso, donde lo propio del convivir consistía en la presión de unos sobre otros. Convivencia era equivalente a compresión. Sin embargo, la presión al ser algo que deriva de aquello que presiona sobre alguien que es presionado conlleva la existencia de un poder, entendido como la dominancia de lo que constituye la realidad. Visto así, lo fundamental no está en la presión sino en el poder, un poder que se funda en el "haber". El haber sería el modo primario del convivir, un problema del co-haber y no de la presión (Zubiri, 2015, pp. 49-51).

Colaboración, estatuto y presión no acaban de explicar en qué consiste el convivir ya que todos ellos son posteriores al "con-vivir" lo que conduce a cuestionar cómo formamos parte de los otro y en qué medida. Aquello en que cada uno es en unidad con los otros y en unidad de los otros con uno. Es en la unidad donde radica la convivencia, lo que da lugar a la pregunta sobre ¿en qué consiste esa unidad? Pero no desde un punto de vista sociológico sino como realidad psico-orgánica, donde tienen lugar tres funciones inseparables: la organización cuya finalidad es dar lugar a la solidaridad de las partes y con ello, hacer manifiesta la corporeidad social. Convivir es que la vida del "yo" tome cuerpo en la vida de los demás; convivir es estar vitalmente presente a los demás, formar cuerpo con ellos. Es una presencia co-presencial, el cuerpo de todos con uno y el de uno con los otros, como uno y único (Zubiri, 2015, pp. 51-56).

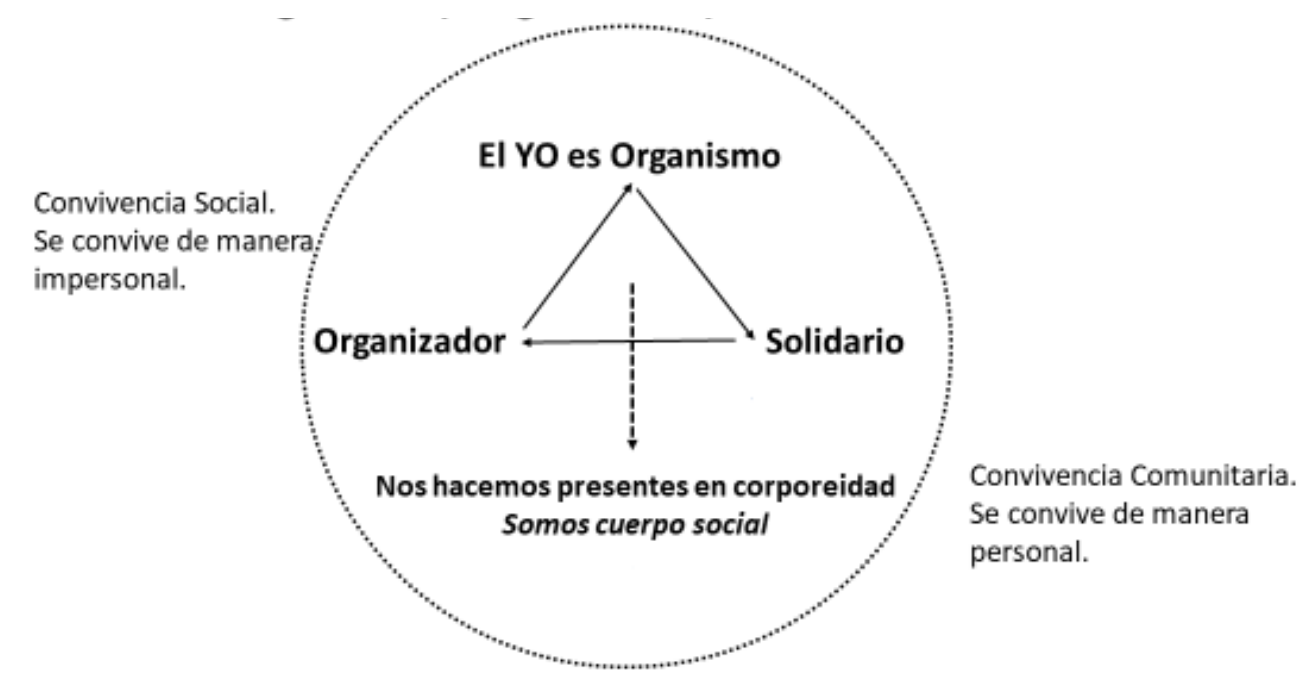

CONVIVIR consiste en que mi vida tome cuerpo en la vida de los demás y se haga presente.

Fig. 3. Convivencia como Organismo / Organizador / Solidario 
Este único cuerpo es la convivencia; el ser humano es en su propia estructura un cuerpo convivencial (Figura 3).

\section{- Cómo nos hacemos presentes}

La manera en que nos vamos haciendo presentes con los otros es en gran medida resultado de las prácticas de la vida cotidiana, lo que tiende a estar modulado por paradigmas, como lo expresa Bernardo Toro (2014). Los paradigmas permiten establecer modos de relación sin mediar necesariamente una reflexión permanente de nuestros actos, situación que generaría excesiva inmovilidad. Sin embargo, el tomar consciencia de los paradigmas que se han heredado y asumido en algún momento de la vida, esto permite descubrir su valor, sentido, trascendencia y con ello la posibilidad de modificación hacia una realización humana más plena.

En la cultura occidental y neoliberal, el paradigma imperante se basa en el éxito, donde hay que poner en juego un poder basado en transacciones, donde la persona tiene como objetivo absoluto el ganar dando lugar a la pérdida la cual es sufrida por otros. Ganar-perder ha sido el paradigma puesto en práctica que en su origen y en algunos momentos de la vida ha sido necesaria como modo de sobrevivencia. El problema radica en que este modo de actuar se ha generalizado a muchas de las dimensiones de nuestra vida cotidiana produce con ello, como señala Toro (2014), una dinámica de acumulación que da lugar a una mayor prisa, competencia, violencia, exclusión, y destrucción de lo que nos rodea. Lo que termina por poner en peligro a la especie humana. Este es el paradigma a cuestionar y para poderlo dimensionar y con ello poner tomar, en un primer momento, decisiones conscientes de nuestros actos, lo que hace no solo necesaria sino urgente una reflexión de carácter ético.

\section{- Un enfoque ético distinto}

La ética implica la reflexión personal como acto intransferible para valorar los actos que tienen lugar en contexto específicos o en el conjunto de la vida. Esto permite que la persona pueda tomar una posición diferente para consigo mismo y para con los demás, y con ello, orientar sus acciones a una nueva dirección. Mientras no se dé esa reflexión seguirán operando, de manera consciente o inconsciente, la inercia de nuestros actos previos. Sin embargo, tampoco podemos afirmar que el vislumbrar un nuevo camino para nuestra acciones y actuaciones se dará de manera inmediata. Esto implica una ruptura de lo cotidiano hacia el cultivo de una nueva práctica que no es fácil de lograr, por simple que parezca.

La ética que ha prevalecido en nuestra cultura ha sido la del deber y que ha derivado en la llamada ética de responsabilidad («Ética de la responsabilidad- Filosofia.mx», 2009), la cual rige los modos de proceder de las instituciones que operan en nuestras sociedades. Es una ética basa en lo que se "debe hacer" como responsabilidad social acorde a la naturaleza de las instituciones en que se asume con el fin de lograr los resultados que se plantean. La ética de la responsabilidad no deja de ser un resultado básicamente racional donde las emociones y sentimientos individuales tienden a ser algo más ajenos. En cambio, la ética del cuidado se basa en una actitud cotidiana de sensibilidad para con los otros y para con uno mismo. Parte de reconocer a la persona como un ser necesitado a lo largo de toda su vida.

La ética del cuidado (Toro \& Boff, 2009) se enfoca en saber com-padecerse con el otro, poder experimentar no solo los dolores sino también los sueños y esperanza que mueven a los sujetos que los rodean. Cuando la persona logra hacer 
presente su compasión, la noción de responsabilidad cobra sustento como algo que permite la implicación integral de la persona, haciendo que la responsabilidad sea un resultado y no un punto impositivo de partida para la actuación.

La ética de la responsabilidad frente a la ética del cuidado son contradictorias en la medida en que se diluyen los aspectos emocionales o racionales de la persona, dando lugar a que las responsabilidades que en un momento se pretenden asumir, pasan a un segundo plano por la fuerza de las pasiones que la persona experimenta o viceversa, que las razones quedan desajustadas de las emociones al carecer de un diálogo interior que permita su articulación.

Partir como principio orientador de la ética del cuidado más que la de responsabilidad nos lleva a incluir lo que emocionalmente vale la pena asumir de una manera responsable. Esta postura implica reconocernos necesitados de los otros e irnos integrando más con ellos y con lo que nos rodea.

En la investigación antes mencionada (Perales et al, 20014), al analizar los cuatro núcleos socio-emocionales validados, éstos se ubicaron con base en sus orientaciones a una de las dos tendencias éticas. Por una parte, los núcleos referidos al respeto y a la responsabilidad se ubican con mayor claridad en el campo de la ética la responsabilidad. Las acciones que ahí se incluyen tienen como característica la de ser acuerdos, sociales o de la cultura del trabajo; acciones y actuaciones verificables ante otros y más fácilmente observables.

Por su parte, los núcleos referidos a la confianza y el cuidado en sus manifestaciones externas, no resultan tan evidentes como son el respeto y responsabilidad; requieren de una finura mayor para develarlas. Se llegan a manifestar un poco más cuando las personas comparten temas que posibilitan la ayuda mutua, ya sea como escucha o como solicitud de apoyo ante diversas circunstancias, así como el poder expresar lo que se piensa sin con ello pretender dañar al otro. Implica también el arte de saber ir y venir respecto del otro para no quedar atrapado en una voluntad ajena. Confianza y cuidado influyen en el desarrollo de un auténtico sentido de respeto y responsabilidad, más allá de una mera formalidad externa (Figura 4).

\section{Provención ${ }^{5}$, un diferente punto de acción}

Un modo para abordar la convivencia en la vida cotidiana desde una visión ética del cuidado tiene que ver con la provención, la que a diferencia de la prevención implica procesos previos, de atención, así como de los recursos necesarios para detectar en el día a día las situaciones de conflicto latentes (Figura 5).

La "prevención" implica tener una visión integral de la situación considerando la dimensión humana, conocimiento de los aspectos estructurales para hacer los cambios necesarios, crear un clima de relaciones cooperativas, basados en el aprecio y la confianza, con canales de comunicación efectiva que permitan generar la conformación de grupos de cooperación, donde se logran separar los problemas de las personas y con todo ello, dando lugar una creciente toma de decisiones por consenso (Gascón, s. f., pp. 12-17).

De esta manera, la provención se ajusta más a una ética del cuidado ya que al poner atención a las relaciones de la vida cotidiana, permite entrelazar hilos de relación que, aparentemente débiles en su forma aislada, terminan por lograr

\footnotetext{
${ }^{5}$ Término acuñado por Burton (1990).
} 


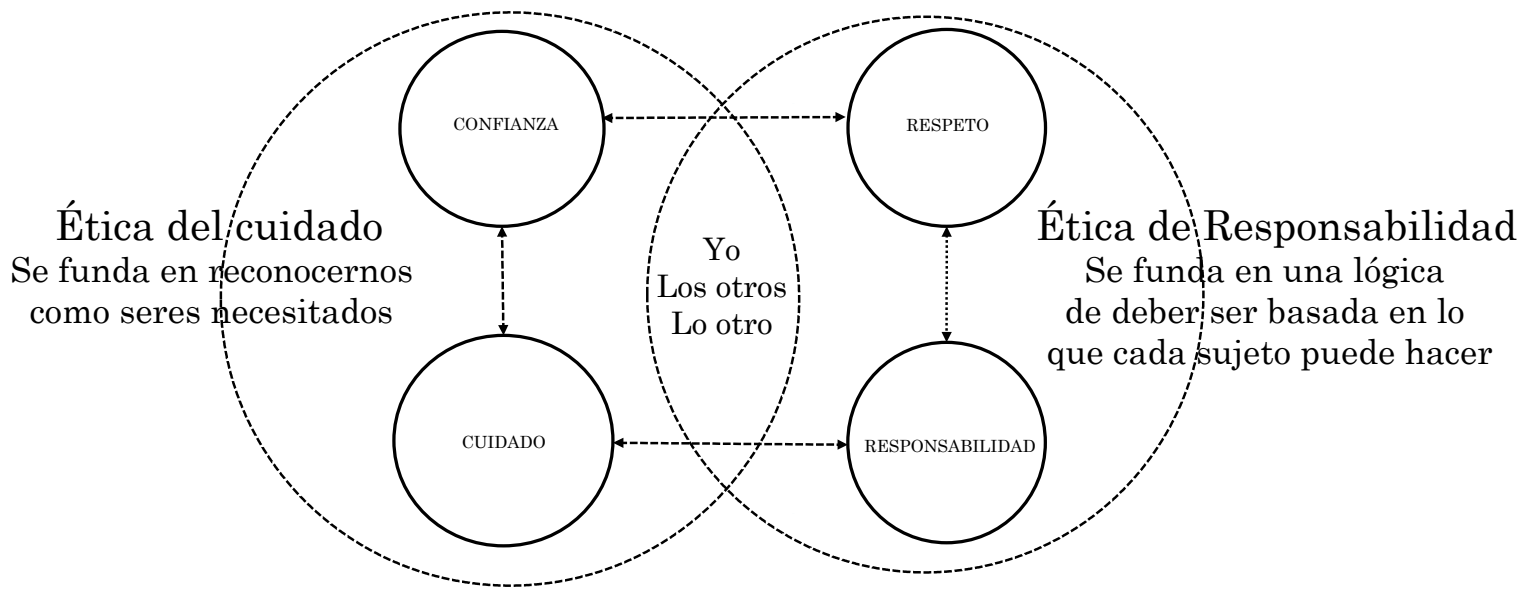

Fig. 4. Ética del Cuidado / Ética de la Responsabilidad

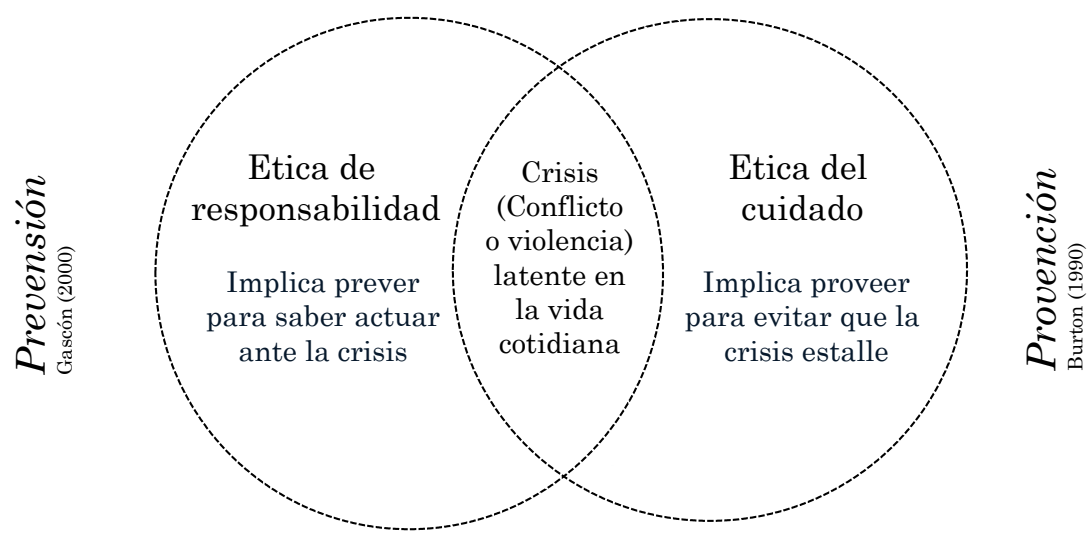

Fig. 5. Cultura de Prevención / Cultura de Provención

de manera casi inadvertida un tejido extenso y resistente ante las variadas tensiones y conflictos latentes que se experimenta en toda relación interpersonal.

\section{Conclusiones}

Delors no estuvo equivocado al vislumbrar a la convivencia como el pilar más importante y necesario para la educación de un futuro que ya es hoy. La riqueza de las reflexiones posteriores se podrá ver profundizadas al abordar el tema no como un mero concepto, sino como parte de lo que constituye la corporeidad del ser humano presente ante los otros, y con ello seguir haciendo presente "el tesoro que todos llevamos dentro". Esto permitirá entender con un poco más de profundidad, las distintas formas de convivencia que nutren la pisco-organicidad del ser humano en contexto específicos, no idealizados, y con ello dar lugar a procesos de aprendizaje diversos que respondan más atinadamente tejer en la cotidianidad interacciones más humanas y humanizantes. 


\section{Referencias}

Bokova, I. (2015). Replantear la educación ¿̇Hacia un bien común mundial? UNESCO.

Burton, J. (1990). Conflict: Resolution and Provention. Virginia: Center Conflict Analysis and Resolution, George Mason University,: The Macmillan Press.

Delors, J., Amagi, I., Carneiro, R., Chung, F., Geremek, B., Gorham, W., ... Savané, K. S. (1997). La educación encierra un tesoro: informe para la UNESCO de la Comisión Internacional sobre la Educación para el Siglo Veintiuno. Unesco.

Filosofia.mx. (2009). Ética de la responsabilidad. (Documento Online). Recuperado de: http://www.filosofia.mx/index.php/perse/archivos/ etica_de_la_responsabilidad

Furlán, A., \& Spitzer, T. C. (2013). Convivencia, disciplina y violencia en las escuelas 2002-2011. México: ANUIES : Consejo Mexicano de Investigación. Recuperado de: http:// www.academia.edu/8260456/PRESENTACION_ESTADO_DEL_CONOCIMIENTO
Hobbes, T. (1994). Leviatán o la materia, forma y poder de una república eclesiástica y civil. México: Fondo de Cultura Económica.

Perales, C., Arias, E., \& Bazdresch, M. (2014). Desarrollo socioafectivo $y$ convivencia escolar. Tlaquepaque, Jalisco: ITESO.

Rousseau, J.-J. (2007). Contrato social. Madrid: Espasa-Calpe.

Toro, B. (2014). El cuidado como paradigma. Recuperado de: https:// www.las 2orillas.co/wp-content/ uploads/2014/11/EL-CUIDADOCOMO-PARADIGMA.pdf. Recuperado 31 de octubre de 2017.

Toro, B., \& Boff, L. (2009). Saber cuidar: El nuevo paradigma ético de la nueva civilización. Elementos conceptuales para una conversación.

UNESCO. (1972). Informe Aprender a Ser. Unesco. Recuperado de: https:// es.scribd.com/doc/107984950/Informe-Aprender-a-Ser-Unesco-1972

Zubiri, X. (2015). Tres dimensiones del ser humano: Individual, Social, Histórica. Fundación Xavier Zubiri. Madrid: Alianza Editorial. 